\title{
S100A2 is a BRCA1/p63 coregulated tumour suppressor gene with roles in the regulation of mutant p53 stability
}

\author{
NE Buckley ${ }^{*, 1}$, Z D'Costa ${ }^{1}$, M Kaminska ${ }^{1}$ and PB Mullan ${ }^{1}$
}

Here, we show for the first time that the familial breast/ovarian cancer susceptibility gene, BRCA1, along with interacting $\triangle$ Np63 proteins, transcriptionally upregulate the putative tumour suppressor protein, S100A2. Both BRCA1 and $\triangle N$ p63 proteins are required for S100A2 expression. BRCA1 requires $\triangle$ Np63 proteins for recruitment to the S100A2 proximal promoter region, while exogenous expression of individual $\triangle \mathrm{Np} 63$ proteins cannot activate $\mathrm{S} 100 \mathrm{~A} 2$ transcription in the absence of a functional BRCA1. Consequently, mutation of the $\triangle N$ p63/p53 response element within the S100A2 promoter completely abrogates the ability of BRCA1 to upregulate S100A2. S100A2 shows growth control features in a range of cell models. Transient or stable exogenous S100A2 expression inhibits the growth of BRCA1 mutant and basal-like breast cancer cell lines, while short interfering RNA (siRNA) knockdown of S100A2 in non-tumorigenic cells results in enhanced proliferation. S100A2 modulates binding of mutant p53 to HSP90, which is required for efficient folding of mutant p53 proteins, by competing for binding to HSP70/HSP90 organising protein (HOP). HOP is a cochaperone that is required for the efficient transfer of proteins from HSP70 to HSP90. Loss of S100A2 leads to an HSP90-dependent stabilisation of mutant p53 with a concomitant loss of p63. Accordingly, S100A2-deficient cells are more sensitive to the HSP-90 inhibitor, 17- $\mathrm{N}$-allylamino-17-demethoxygeldanamycin, potentially representing a novel therapeutic strategy for S100A2- and BRCA1-deficient cancers. Taken together, these data demonstrate the importance of S100A2 downstream of the BRCA1/ $\triangle \mathrm{Np63}$ signalling axis in modulating transcriptional responses and enforcing growth control mechanisms through destabilisation of mutant $\mathrm{p} 53$.

Cell Death and Disease (2014) 5, e1070; doi:10.1038/cddis.2014.31; published online 20 February 2014

Subject Category: Cancer

BRCA1 was initially cloned in 1994 as one of the genes predisposing to early-onset breast and ovarian cancer. ${ }^{1}$ BRCA1 germline mutations confer a cumulative lifetime risk of $50-85$ and $12-60 \%$ of developing breast and ovarian cancer, respectively. ${ }^{2}$ Somatic BRCA1 mutations are rare in sporadic breast cancer, but BRCA1 expression is downregulated in $\sim 30 \%$ of sporadic cases. ${ }^{3}$ BRCA1 is known to have multiple roles including DNA damage repair, cell cycle checkpoint control and transcriptional regulation. Although BRCA1 does not bind to DNA in a sequence-specific manner, it facilitates transcriptional control at a number of different levels (e.g. interacts with transcription factors, the RNA polymerase II holoenzyme complex and proteins involved in chromatin remodelling; for a review see Mullan et al. ${ }^{4}$ ). Through these multiple interactions, BRCA1 can coactivate or corepress a large number of target genes involved in its downstream functions.

Our group has recently identified the $\Delta$ Np63 family of proteins as novel BRCA1 interactors with important roles in BRCA1-mediated tumour suppression. ${ }^{5}$ Our data suggest that the transcriptional control of $\Delta \mathrm{Np} 63$ expression by
BRCA1 is important for the maintenance of genomic stability and for the normal differentiation of mammary tissue. The tumour suppressor role of p63 in breast tissue was also demonstrated by the finding that mutant p53 proteins may drive tumourigenesis through the formation of p53-SMAD complexes, which interacts with and opposes p63 function, leading to enhanced metastasis. ${ }^{6}$

The family of $\mathrm{S} 100$ proteins is a highly conserved group of more than 20 members of small $(9-13 \mathrm{kDa})$ acidic calciumbinding proteins. ${ }^{7}$ Numerous $\mathrm{S} 100$ proteins are known to be overexpressed in cancers, while S100A2 is thought to primarily act in a tumour-suppressive role. $^{8-10}$ S100A2 expression is downregulated in several tumour types including breast cancer, ${ }^{11}$ with promoter methylation being one mechanism responsible for this. ${ }^{9}$ A number of $\mathrm{S} 100$ proteins have been shown to interact with the p53 tumour suppressor, interactions that may be influenced through p53 phosphorylation and acetylation events, potentially activating as well as inhibiting p53. ${ }^{12}$ Specifically, S100A2 is known to interact with wild-type p53 and other p53 family members to enhance their transcriptional activity. ${ }^{13}$ S100A2 has been shown previously

${ }^{1}$ Centre for Cancer Research and Cell Biology, Queen's University Belfast, Belfast, UK

${ }^{*}$ Corresponding author: NE Buckley, Centre for Cancer Research and Cell Biology, Queen's University Belfast, 97 Lisburn Road, Belfast BT9 7BL, UK. Tel: +44 (0)28 90975806; Fax: +44 (0)28 90972776; E-mail: n.obrien@qub.ac.uk

Keywords: BRCA1; S100A2; mutant p53

Abbreviations: 17-AAG, 17- N-allylamino-17-demethoxygeldanamycin; 17-DMAG, 17-dimethylaminoethylamino-17-demethoxygeldanamycin; BrdU, bromodeoxyuridine; ChIP, chromatin immunoprecipitation; EV, empty vector; HOP, HSP70/HSP90-organising protein; MTT, 3-(4,5-dimethylthiazol-2-yl)-2,5-diphenyltetrazolium bromide; TPR domain, tetratricopeptide repeat domains

Received 01.10.13; revised 13.1.14; accepted 15.1.14; Edited by G Raschellà 
to be a direct transcriptional target of both p63 and p73 proteins. ${ }^{14,15}$ The function of S100A2 (also called S100L) is still unknown but it is localised to the basal layer of normal human epidermis and hair follicles, possibly reflecting its p63dependent activation. ${ }^{16,17}$

In this study, we show that $\mathrm{S} 100 \mathrm{~A} 2$ is a coregulated target of both $B R C A 1$ and $\triangle N p 63$, requiring both proteins to be expressed and fully functional for optimal S100A2 expression. We observe consistent growth inhibitory effects in multiple breast cancer cell lines and non-tumourigenic breast cell lines, consistent with its role as a tumour suppressor in breast tissue. S100A2 knockdown results in an increase in mutant p53 with a concomitant loss of p63. We demonstrate that the observed increase in p53 is owing to HSP90-dependent stabilisation and S100A2-depleted cells are therefore more sensitive to the HSP90 inhibitor, 17- N-allylamino-17demethoxygeldanamycin (17-AAG). Taken together, these data suggest a role for a BRCA1- $\triangle$ Np63-S100A2 signalling pathway important for the growth control of breast tissue through the regulation of HSP90-client protein interaction.

\section{Results}

We have consistently observed $\mathrm{S} 100 \mathrm{~A} 2$ as a positively regulated $B R C A 1$ target gene following several microarray experiments (data not shown). To validate this, we first stably reconstituted wild-type BRCA1 into the BRCA1 mutant HCC1937 and basal-like (BRCA1 low-expressing) MDA-MB468 cells. Figure 1ai shows western blot analyses of HCC1937-BR cells showing marked upregulation of S100A2 protein following $\mathrm{BRCA} 1$ reconstitution relative to the empty vector (EV) control cell line, HCC1937-EV. Figure 1aii confirmed that this effect was transcriptional with an approximate fivefold upregulation of S100A2 mRNA. Similar effects were observed with MDA-MB-468 cells relative to EV controls (Figures 1 bi and ii). In contrast, siRNA knockdown of BRCA1 in non-tumourigenic HME-1 (Figures 1ci and ii) or luminal MCF7 cells (Figures $1 \mathrm{di}$ and ii) resulted in respective downregulation of S100A2 proteins and mRNAs. S100A2 was also shown to be downregulated in BRCA1-associated tumours using publically available data sets (Supplementary Figure 1). Clearly, S100A2 is regulated by BRCA1 at a protein and mRNA level in multiple breast cell lines and in primary breast cancers.

As detailed in the Introduction, we recently identified the $\triangle \mathrm{Np63}$ family of proteins as BRCA1 transcriptional targets. ${ }^{5}$ BRCA1 transcriptionally regulates these proteins through specific interaction with $\Delta \mathrm{Np} 63 \gamma$ to drive a positive $\Delta \mathrm{Np} 63$ regulatory loop. S100A2 has already been described as a p63 target gene, ${ }^{14,15}$ so we decided to investigate the mechanism underpinning S100A2 upregulation. Both HCC1937- and MDA-MB-468 BRCA1-reconstituted cell lines were treated (alongside EV controls) with $\triangle$ Np63siRNA. As Figures $2 \mathrm{a}$ and b show, we observed strong induction of $\triangle \mathrm{Np63}$ in BRCA1reconstituted cells (relative to $\mathrm{EV}$ controls) accompanied by pronounced upregulation of S100A2. However, $\triangle$ Np63 siRNA totally abrogated $\mathrm{S} 100 \mathrm{~A} 2$ protein and mRNA in BRCA1reconstituted cells, showing that the $B R C A 1-\Delta N p 63$ complex is a crucial regulator of $\mathrm{S} 100 \mathrm{~A} 2$. To demonstrate the $\triangle \mathrm{Np} 63$ specificity, we performed $\Delta$ Np63-, TAp63- and p53-specific
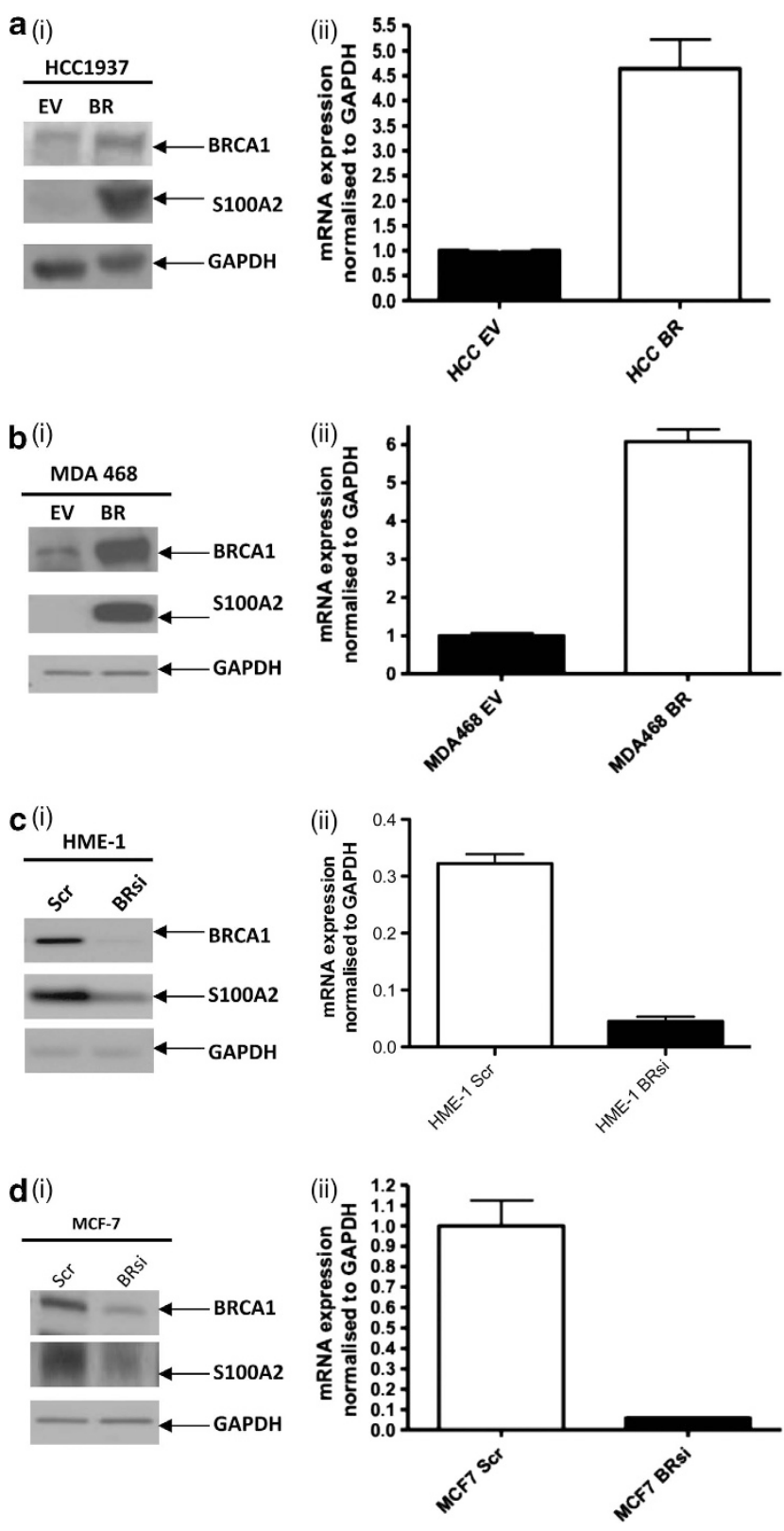

Figure 1 Western blot analysis of (ai) BRCA1 mutant HCC1937 and (bi) BRCA1 low MDA468 (MDA-MB-468) breast cancer cells, stably transfected with EV or wild-type BRCA1 (BR). Blots were probed with BRCA1, S100A2 and glyceraldehyde 3-phosphate dehydrogenase (GAPDH) as a loading control. Real-time polymerase chain reaction (PCR) analysis of (aii) HCC EV and BR and (bii)) MDA468 EV and BR cells with primers specific for S100A2. GAPDH was used as a housekeeper. Western blot analysis of (ci) HME1 and (di) MCF7 breast cancer cells transiently transfected with siRNA targeting BRCA1 (BRsi) or scrambled control (Scr). Blots were probed with BRCA1, S100A2 and GAPDH as a loading control. (cii and dii) Real-time PCR analysis of the same samples as before with primers specific for S100A2. GAPDH was used as a loading control

siRNA knockdowns in MCF7 cells (Supplementary Figure 2A) and immunoblotted for S100A2. As Figure 2ci shows, only $\triangle \mathrm{Np63}$ knockdown reduced S100A2 levels and this was consistent for $\gamma$ - or $\alpha / \beta$-specific siRNAs (Figure 2cii, knockdowns shown in Supplementary Figure 2Bi). Similar results are also seen with knockdown of mutant p53 in the MDA-BR 
cell lines (Supplementary Figure 2C). p63 often transcriptionally activates basal target genes in collaboration with the AP2 family of proteins. ${ }^{18}$ We were able to show that AP $2 \alpha$ was also required for S100A2 activation (Figure 2cii, knockdowns shown in Supplementary Figures 2Bii and iii). Finally, we decided to investigate if exogenous expression of p63 could bypass the requirement for BRCA1 expression and activate S100A2 independently. BRCA1 mutant HCC1937 cells were transfected with either an EV construct or different $\triangle \mathrm{N}$ - or TAp63 isoforms. As Figure $2 d$ shows, only BRCA1 reconstitution restored S100A2 expression, suggesting that for S100A2 promoter activation, BRCA1 was still required regardless of p63 levels. These data together show that both BRCA1 and $\triangle$ Np63 proteins (in collaboration with AP2 $\alpha$ ) are required for optimal activation of S100A2 transcription.

Next, we wanted to define exactly how BRCA1 modulated S100A2 promoter activity. We PCR cloned the proximal S100A2 promoter and transfected into a variety of cell lines with modulated BRCA1. For both HCC1937- and MDA-MB468 BRCA1-reconstituted cells, we observed significant
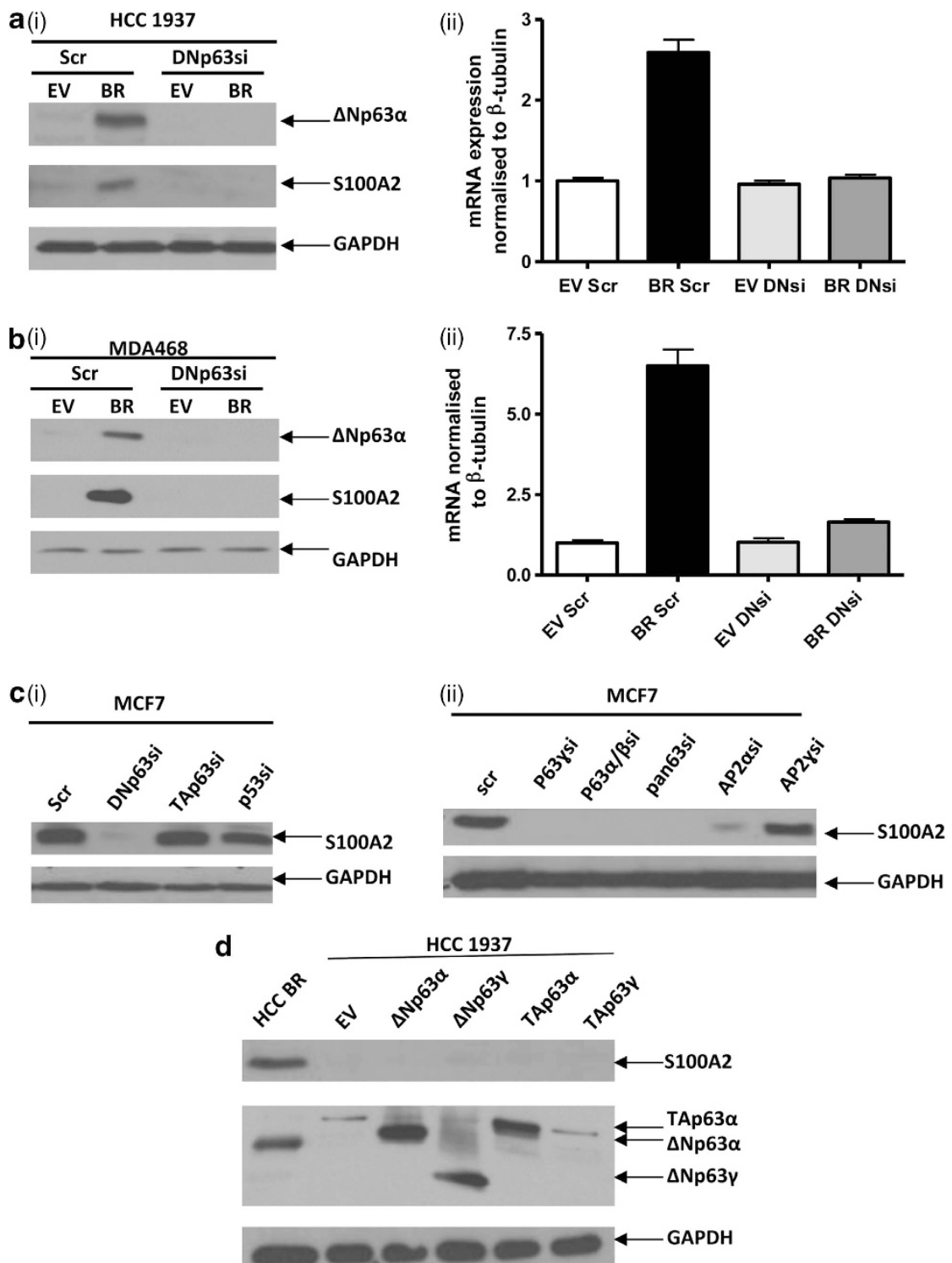

Figure 2 (ai) Western blot analysis of BRCA1 mutant HCC 1937 cells stably transfected with either EV or wild-type BRCA1 (BR) and transiently transfected with siRNA targeting $\triangle \mathrm{Np} 63$ (DNp63si) or scrambled control (Scr). Blots were probed with p63, S100A2 and glyceraldehyde 3-phosphate dehydrogenase (GAPDH) as a loading control. (aii) Real-time polymerase chain reaction (PCR) analysis of the same samples as (i) with specific primers for S100A2 and $\beta$-tubulin as a housekeeper. (bi) Western blot analysis of BRCA1 low MDA468 cells stably transfected with either EV or wBR and transiently transfected with siRNA targeting $\triangle$ Np63 (DNp63si) or Scr. Blots were probed with p63, S100A2 and GAPDH as a loading control. (bii) Real-time PCR analysis of the same samples as (i) with specific primers for S100A2 and $\beta$-tubulin as a housekeeper. (ci) Western blot analysis of MCF-7 cells transiently transfected with siRNA targeting $\triangle$ Np63 (DNp63si), TAp63 (TAp63si), p53 (p53si) or Scr. Blots were probed with S100A2

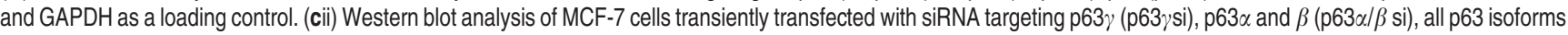
(pan-p63), AP2 $\alpha$ (AP2 $\alpha$ si), AP2 $\gamma$ (AP2 $\gamma$ si) and Scr. Blots were probed with S100A2 and GAPDH as a loading control. (d) Western blot analysis of HCC BR cells or HCC 1937 cells transiently transfected with EV, $\Delta \mathrm{Np} 63 \alpha, \Delta \mathrm{Np} 63 \gamma$, TAp63 $\alpha$ or TAp63 $\gamma$. Blots were probed for S100A2, p63 and GAPDH as a loading control 
luciferase activities that were markedly reduced with siRNA against $\triangle$ Np63 (Figures 3 ai and ii). Similarly, BRCA1 and $\triangle$ Np63 siRNA knockdowns in MCF7 cells reduced S100A2 luciferase, while p53 knockdown had minimal effect (Figure 3aiii). In addition to the previously characterised p53 binding site located $\sim 2200$ bp upstream of the untranslated exon 1 of the S100A2 gene, ${ }^{19}$ Kirschner et al. ${ }^{15}$ described a second p63-specific binding site located just upstream of the first exon. While deletion of the p53-responsive site had no effect on the observed luciferase activity, we could demonstrate that the predicted $\Delta \mathrm{Np} 63$ binding site was responsible for this activation since site-directed mutagenesis of this site completely abrogated the BRCA1-dependent luciferase activation in both HCC1937 and MDA-MB-468 cell models
(Figures 3bi and ii, respectively). We also PCR cloned two regions within intron 2 of $S 100 A 2$, which have been shown to be p63 responsive. ${ }^{14}$ Although these regions were p63 responsive, they did not appear to be consistently regulated by BRCA1 (Supplementary Figure 3); therefore, these promoter regions were not studied further. Chromatin immunoprecipitation (ChIP) assays showed localisation of both BRCA1 and $\triangle \mathrm{Np} 63$ to the S100A2 proximal promoter region, while an upstream $(-2 \mathrm{kB}$ ) region showed no obvious recruitment of either protein (Figure 3ci). Finally, we could demonstrate the requirement for $\triangle \mathrm{Np} 63$ for BRCA1 recruitment to the S100A2 promoter using $\triangle N$ p63 siRNA knockdown followed by ChIP assay, showing total loss of BRCA1 recruitment following $\triangle$ Np63 siRNA (Figure 3cii). Taken
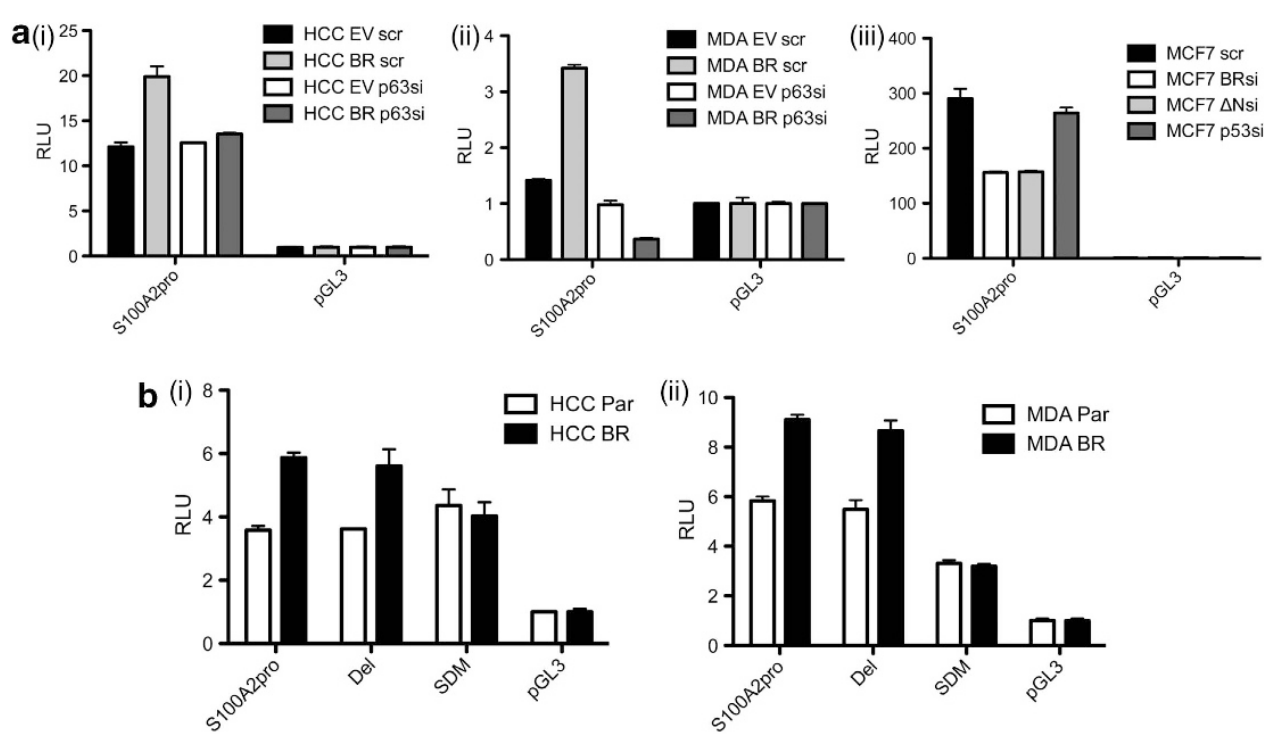

C
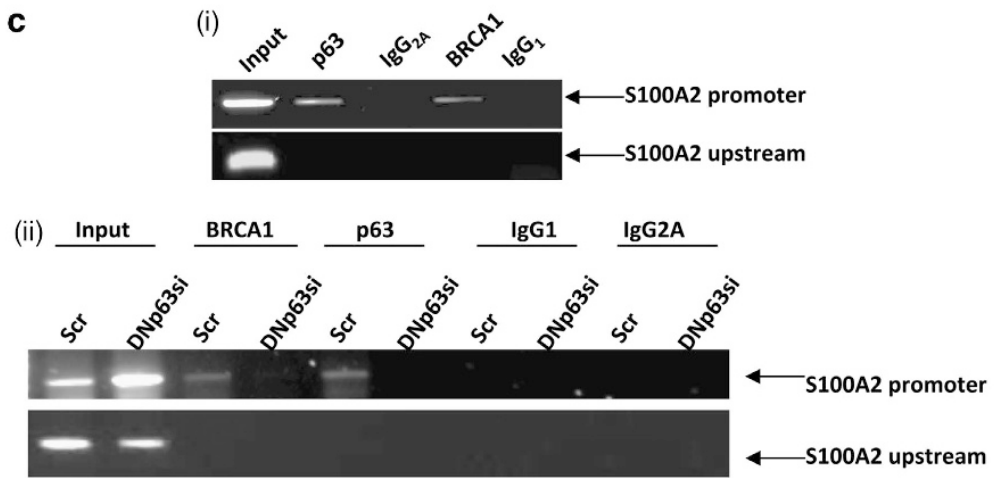

Figure 3 (a) Luciferase reporter assay of (i) HCC EV and wild-type BRCA1 (BR) cells and (ii) MDA EV and BR cells transiently transfected with siRNA targeting $\triangle N p 63$ (p63si) or scrambled control (Scr). Cells were then transfected with either S100A2 promoter construct (S100A2pro) or the EV control (pGL3). Renilla was used to normalise for transfection efficiency. Values are expressed as relative luciferase units (RLUs) normalised to pGL3 and Renilla. (iii) Luciferase reporter assay of MCF7 cells transiently transfected with siRNA targeting BRCA1 (BRsi), $\Delta$ Np63 ( $\Delta$ Nsi), p53 (p53si) or Scr. Cells were then transfected with either S100A2 promoter construct (S100A2pro) or the EV control (pGL3). Renilla was used to normalise for transfection efficiency. Values are expressed as RLUs normalised to pGL3 and Renilla. (b) Luciferase reporter assay of (i) HCC EV and BR cells and (ii) MDA EV and BR cells transfected with either an S100A2 promoter construct (S100A2pro), an S100A2 promoter construct with one p53/p63 binding site deleted (Del), an S100A2 promoter construct with one p53/p63 binding site deleted and the second mutated (SDM) or the EV control (pGL3). Renilla was used to normalise for transfection efficiency. Values are expressed as RLUs normalised to pGL3 and Renilla. (ci) ChIP assay of MDA468 BR cells showing localisation of p63 and BRCA1 on the S100A2 promoter. One per cent of sonicated lysate before immunoprecipitation was used as a positive control (input) and lgG pulldown was used as a negative control ( $\left(g G_{1}\right.$ and $\lg G_{2 a}$ ). In addition, polymerase chain reaction (PCR) primers for a region upstream of the S100A2 promoter show specificity for the promoter. (ii) $C h I P$ assay of MDA468 BR cells transiently transfected with siRNA targeting $\triangle N p 63$ (DNp63si) or Scr showing recruitment of BRCA1 and p63 to the S100A2 promoter. One per cent of sonicated lysate before immunoprecipitation was used as a positive control (input) and $\lg G$ pulldown was used as a negative control (IgG $\mathrm{I}_{1}$ and $\lg \mathrm{G}_{2 \mathrm{a}}$ ). In addition, $P C R$ primers for a region upstream of the S100A2 promoter shows specificity for the promoter 
together, these data demonstrate that $\Delta$ Np63-dependent recruitment to a specific site in the S100A2 promoter is required for its transcriptional activation.

S100A2 has previously been reported to act as a tumour suppressor gene in breast tissue; ${ }^{10}$ thus, we wanted to address the functional consequences of S100A2 loss and how important this protein was for BRCA1-mediated tumour suppression. Stable exogenous S100A2 expression was performed in the BRCA1-defective HCC1937, MDA-MB-468 and transient S100A2 expression in the SUM149 cells (Figures 4ai-ii and Supplementary Figure 4Aii) resulted in a reproducible inhibition of cell growth, as assessed by 5-day growth curves (Figures 4bi-ii and Supplementary Figure $\mathrm{Ai}$ ). Again knockdowns of S100A2 in BRCA1-proficient, non-
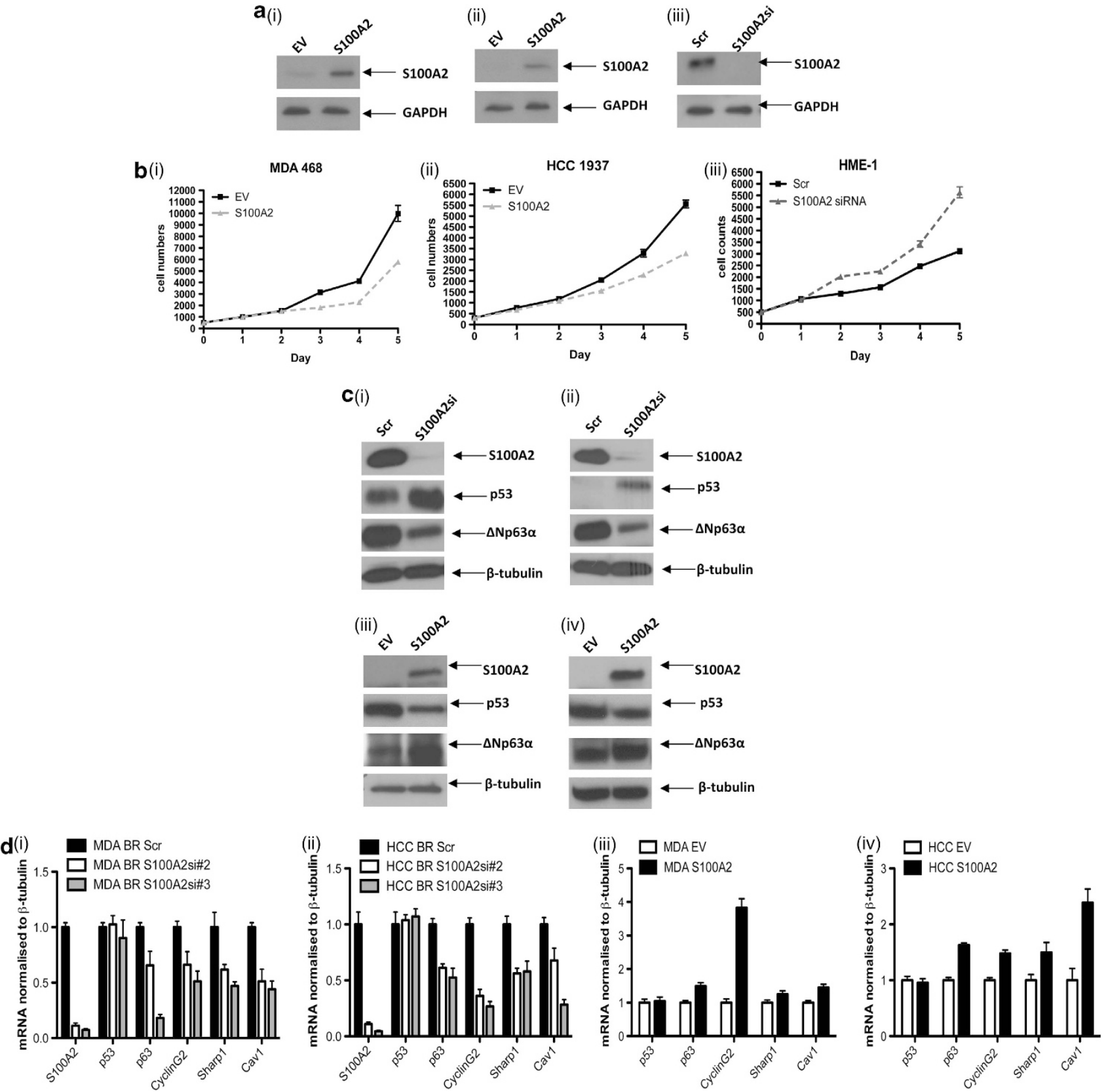

Figure 4 (a) Western blot analysis of (i) MDA468 or (ii) HCC1937 cells stably transfected with either EV or S100A2 or (iii) HME-1 cells transiently transfected with siRNA targeting S100A2 or scrambled control (Scr) probed for S100A2 and glyceraldehyde 3-phosphate dehydrogenase (GAPDH) as a loading control. (b) Five-day growth curve of the same samples as (a). (c) Western blot analysis of (i) MDA BR or (ii) HCC BR cells transiently transfected with siRNA targeting S100A2 or Scr. Blots were probed for S100A2, p53, p63 and $\beta$-tubulin as a loading control. Western blot analysis of (iii) MDA 468 and (iv) HCC 1937 cells stably transfected with either EV or S100A2. Blots were probed for S100A2, p53, p63 and $\beta$-tubulin as a loading control. (d) Real-time polymerase chain reaction (PCR) analysis of (i) MDA BR or (ii) HCC BR cells transiently transfected with two independent siRNAs targeting S100A2 (S100A2 no. 2 and S100A2 no. 3) and Scr using primers specific for S100A2, p53, p63 (pan), CyclinG2, Sharp1 and Caveolin1 (Cav1). Real-time PCR analysis of (iii) MDA 468 and (iv) HCC 1937 cells stably transfected with either EV or S100A2 using primers specific for S100A2, p53, p63 (pan), CyclinG2, Sharp1 and Caveolin1 (Cav1) 
tumourigenic HME1 and MCF10-A cells (Figure 4aiii and Supplementary Figure 4Bii) resulted in enhanced proliferation (Figure 4biii and Supplementary Figure 4Bi). Consistent with previous findings, the effect of S100A2 on cell numbers appears to be through the regulation of transition from the $S$ to G2/M phase of the cell cycle and not through the regulation of cell viability. ${ }^{20}$ Although PARP cleavage (indicative of apoptosis) is not observed when S100A2 is modulated (Supplementary Figure $4 \mathrm{C}$ ), there is an increase in the number of cells in the $S$ phase (as measured by bromodeoxyuridine (BrdU) incorporation) when S100A2 is stably overexpressed in HCC1937 and the opposite seen when S100A2 is depleted by siRNA (Supplementary Figure 4D). An interesting observation was made that while BRCA1 and $\triangle \mathrm{Np63}$ knockdowns resulted in S100A2 downregulation, S100A2 siRNA also resulted in an increase in mutant p53 protein levels with a concomitant reduction in $\triangle \mathrm{Np} 63$ protein levels in both HCC1937-BR and MDA-MB-468-BR cell lines (Figures 4ci-ii). Similar effects were also observed with BRCA1 and $\triangle$ Np63siRNA (Supplementary Figure 5), as well as in the BRCA1 low expression MDA468-EV cells (Supplementary Figure 2C). Using the converse approach of stable overexpression of S100A2 in the BRCA1-deficient MDA-MB-468 and HCC1937 cells, we observed a decrease in mutant p53 protein levels and an increase in $\Delta \mathrm{Np} 63$ protein levels (although $\triangle \mathrm{Np} 63$ levels were considerably lower than those observed in BRCA1-proficient cell lines) (Figures 4ciii and iv). Although the effect on p53 levels was not transcriptional, the effect on $\triangle N p 63$ was clearly evident at an mRNA level (Supplementary Figure 5B). Previous studies have demonstrated that mutant p53, particularly 'gain-of-function' mutant p53 proteins are able to interfere with the tumoursuppressive role of p63 to drive metastasis. ${ }^{6}$ Accordingly, the expression of p63 target genes, CyclinG2, Sharp1 and Caveolin1, were all modulated in an S100A2-dependent manner (Figure 4d). Studies are ongoing to determine the exact mechanism of how S100A2 regulates p63; however, preliminary results (Supplementary Figure $5 \mathrm{C}$ ) indicate it may be dependent on the ability of mutant p53 to oppose p63 function and this would impact on the autoregulation of p63 through the $\mathrm{C} 40$ enhancer region. ${ }^{5,21}$

As S100A2 levels appeared to antagonise mutant p53 expression, we hypothesised that S100A2 may somehow alter the stability of mutant p53 to suppress tumourigenesis. It has been documented that certain p53 missense mutants are dependent on chaperones such as HSP90 for their stability. ${ }^{22}$ Given the ability of S100A2 to interact with the cochaperone HSP70/HSP90 organising protein (HOP) and therefore modulate the transfer of client proteins from HSP70 to HSP $90,{ }^{23}$ we investigated the effect of S100A2 on the chaperone-dependent folding and thus the stability of mutant p53. Levels of mutant p53 in the denatured and native conformation were quantified by immunoprecipitation using specific antibodies (PAb-240 and PAb-1620, respectively) and compared with total levels as determined by immunoprecipitation with an antibody that detects total p53 levels (DO-1). Following knockdown of S100A2 in MDA468-BR cells, total p53 increased as shown previously (Figure 5ai and Supplementary Figure 6Ai). As the majority of the contact mutant $(\mathrm{R} 280 \mathrm{H}) \mathrm{p} 53$ in the MDA-MB-468 cell line is found in the native conformation, ${ }^{22}$ it was only possible to compare the overall levels of mutant p53. Knockdown of S100A2 resulted in an increase in the native form of mutant p53, normalised to total p53 protein (Figure 5aii and Supplementary Figure 6Ai). Conversely, stable overexpression of S100A2 in the HCC1937 cells resulted in the expected decrease in total p53 (Figure 5bi and Supplementary Figure 6Aii) with a decrease in the native form and an increase in the denatured form of mutant p53 (Figures 5bii and iii and Supplementary Figure 6Aii). To determine if S100A2 modulated the binding of mutant p53 to HSP90, we assessed the amount of denatured mutant p53 interacting with HSP90 by immunoprecipitation. In MDA468-BR cells, knockdown of S100A2 resulted in an increase in denatured p53 interacting with HSP90 (Figure 5ci). Conversely, overexpression of S100A2 in the HCC1937 cell led to a decrease (Figure 5cii). We confirmed that S100A2 interacted with HOP (but not HSP90) by immunoprecipitation (Figure 5di) and demonstrated that loss of S100A2 expression results in increased HOP-HSP90 interaction (Figure 5dii) and therefore predicts for increased client protein transfer from HSP70 to HSP90. Next, we utilised the HSP-90 inhibitor, 17-AAG, to evaluate the dependency of mutant p53 for HSP90-mediated folding. Knockdown of S100A2 in MDA468-BR cells led to the expected increase in mutant $\mathrm{p53}$, but this was abrogated by treatment with 17-AAG (Figure 5ei). These cells were also more sensitive to the inhibitor (relative to scrambled control siRNA-treated cells) (Figure 5fi and Supplementary Table 1). Similar results are also seen in the HCCBR and HME-1 cells following siRNA (Supplementary Figure 6B and Supplementary Table 1),

\footnotetext{
Figure 5 (a) Quantitation by densitometry of immunoprecipitation (IP) in MDA BR cells transiently transfected with two independent siRNAs targeting S100A2 (S100A2 no. 2 and S100A2 no. 3) and scrambled control (Scr). (i) Total p53 was immunoprecipitated using D01 and (ii) folded/native conformation p53 was immunoprecipitated using pAB1620. (b) Quantitation by densitometry of IP in HCC 1937 cells stably transfected with EV or S100A2. (i) Total p53 was immunoprecipitated using DO1, (ii) folded/native conformation p53 was immunoprecipitated using pAB1620 and (iii) denatured p53 was immunoprecipitated using pAB240. (c) Co-immunoprecipitation of (i) MDA BR cells transiently transfected with siRNA targeting S100A2 (SA2si) and Scr and (ii) HCC1937 cells stably transfected with EV or S100A2 (SA2). Cell lysates were immunoprecipitated with pAB240, and then following western blot analysis, probed for HSP90. (di) IP-western blot analysis of MDA BR cells. Whole-cell lysate was immunoprecipitated with either S100A2 antibody or an IgG control followed by western blot analysis. Blots were probed with HSP90, HOP or S100A2. (ii) IP-western blot of MDA BR cells was transiently transfected with two independent siRNAs targeting S100A2 (S100A2 no. 2 and S100A2 no. 3) and Scr. Whole-cell lysate was immunoprecipitated with either HSP90 or IgG control followed by western blot analysis. Blots were probed for HSP90, HOP and S100A2. (ei) Western blot analysis of MDABR cells transiently transfected with two independent siRNAs targeting S100A2 (SA2si no. 2 and no. 3) or Scr. Cells were then treated with $4 \mu \mathrm{M}$ 17-AAG or vehicle control for $16 \mathrm{~h}$. Blots were probed with p53, $\mathrm{S} 100 \mathrm{~A} 2$ or $\beta$-tubulin as a loading control. (ii) Western blot analysis of HCC1937 cells stably transfected with EV control or S100A2 (SA2). Cells were treated with $2 \mu \mathrm{M} 17-\mathrm{AAG}$

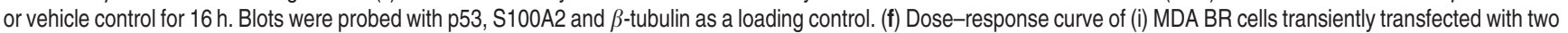
independent siRNAs targeting S100A2 (S100A2 no. 2 and no. 3) and Scr and (ii) HCC1937 cells stably transfected with EV or S100A2 (S100A2). Cells were treated with the indicated range of concentration of $17-\mathrm{AAG}$ for $72 \mathrm{~h}$ before cell viability was assessed by MTT. Cell survival was normalised to vehicle control (100\%)
} 
as well as in HCC1937 cells stably reconstituted with S100A2, with S100A2 conferring over 10-fold decrease in sensitivity to 17-AAG compared with EV control (Figures 5eii and fii and Supplementary Table1). Using cell line data from Lehmann et al., ${ }^{24} \mathrm{IC}_{50}$ values for the 17-AAG analogue, 17-dimethylaminoethylamino-17-demethoxygeldanamycin
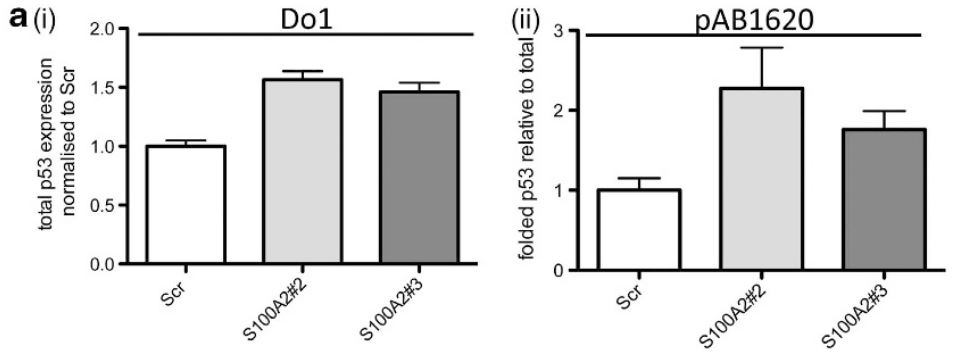

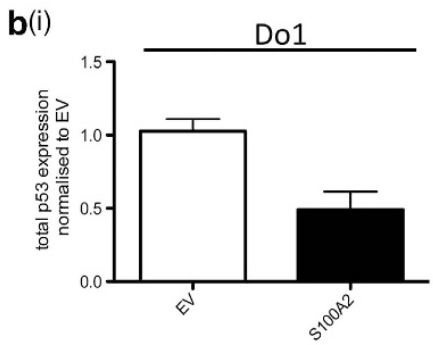

c(i) (ii)

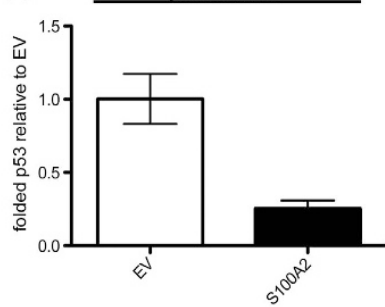

(iii)

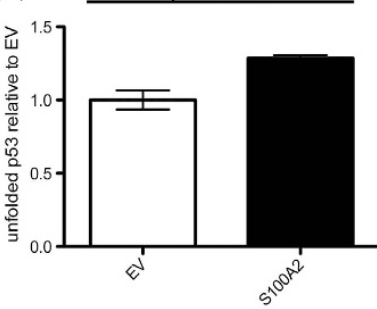

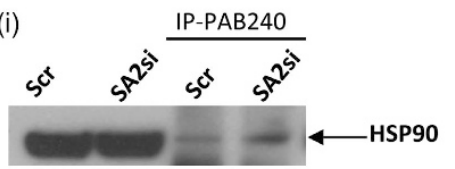

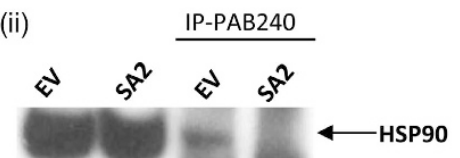

d(i)

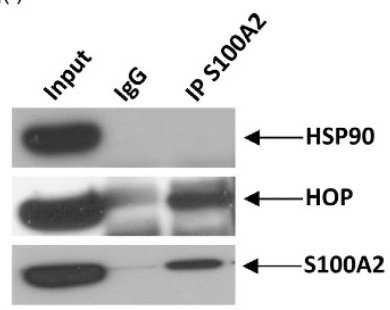

(ii) (ii) Input

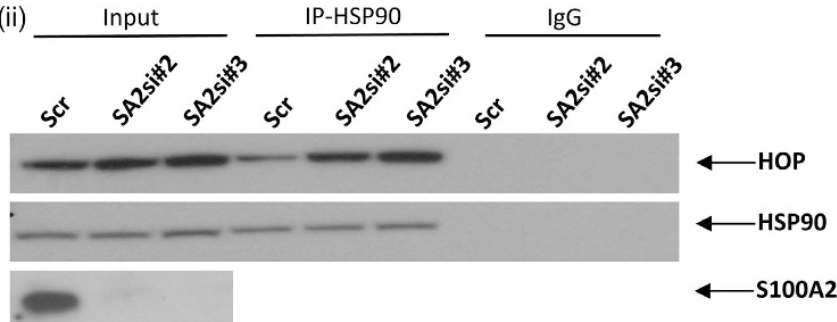

$\mathbf{e}(\mathrm{i})$

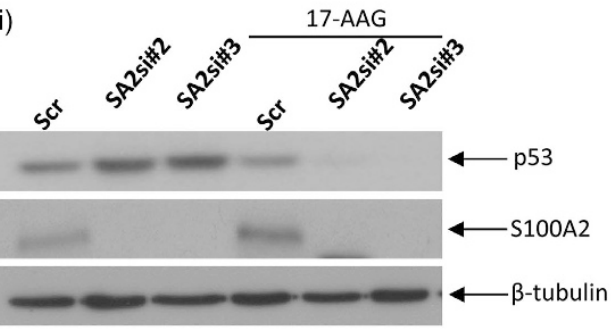

(ii)

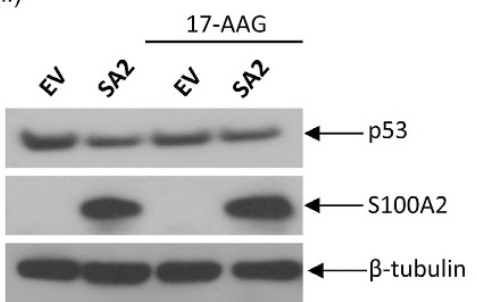

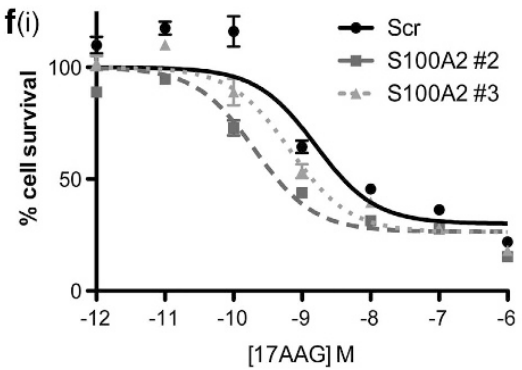

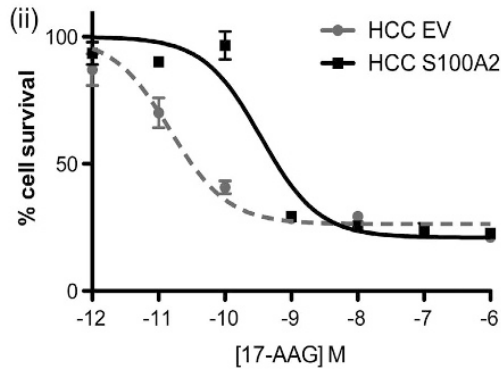


(17-DMAG), were extrapolated and correlation with S100A2 expression levels (as determined by microarray analysis of the same publically available data sets utilised in the study) was assessed (Supplementary Figure 6C and Supplementary Table1). This showed highly significant correlation between S100A2 levels and sensitivity to HSP90 inhibition. Taken together, these data highlight the important role of S100A2 as a regulator of mutant p53 protein levels and sensitivity to HSP90 inhibition.

\section{Discussion}

In this study, we have identified $\mathrm{S} 100 \mathrm{~A} 2$ as an integral component of BRCA1 tumour suppression downstream of the BRCA1- $\triangle$ Np63 transcription complex. We show that S100A2 consistently represses cell proliferation in a range of breast cell lines and that S100A2 expression is doubly important for regulating both the optimal transcription of p63 and for helping to destabilise mutant p53 protein levels. We have shown that S100A2 interferes with the transfer of client proteins from HSP70 to HSP90, therefore inhibiting the folding and stabilisation of the mutant protein. Inhibition of HSP90 abrogates the increase in mutant p53 observed when S100A2 expression is lost and S100A2-deficient cells are acutely sensitive to the HSP90 inhibitor, 17-AAG.

The role of $\mathrm{S} 100 \mathrm{~A} 2$ in tumour suppression is still poorly described. S100A2 is the only member of the $\mathrm{S} 100$ family that appears to act in a tumour-suppressive manner, while the others have oncogenic roles. Of interest, our colleagues have previously identified a number of S100 family members as genes repressed by BRCA1 ${ }^{25}$ The BRCA1-dependent repression of S100A7 (or psoriasin) was shown to have as pivotal role in BRCA1-dependent resistance to the DNA-damaging agent, etoposide. $^{25}$ Our data show that S100A2 is an important effector of the BRCA1/p63 tumour-suppressive pathway that we have described previously. ${ }^{5}$ The observation that loss of S100A2 leads to a downregulation of p63 means that any mechanism leading to a loss of S100A2 expression, such as promoter methylation, may result in a loss of the BRCA1/p63 tumour suppressor function leading to tumourigenesis.

Much of the interest into the role of S100A2 as a tumour suppressor relates to its coactivation of wild-type p53 target genes. ${ }^{13}$ However, this is the first time that S100A2 has been shown to modulate mutant p53 protein stability. Up to $40 \%$ of human cancers express high levels of mutant p53 and $74 \%$ of these are missense mutations that fall within its central DNAbinding domain. ${ }^{26}$ Using both zebrafish and mouse models, studies have shown that mutation alone is not sufficient to confer stability to the protein in the absence of stress. ${ }^{27,28}$ Therefore, tumour-specific activation of p53 stabilisation pathways is required - an area that is still poorly understood.

Initially, it was thought that the increase in stability of mutant p53 was owing to the fact that it did not induce the wild-type p53 target gene, MDM2. However, this is an oversimplified view as mice engineered to express mutant p53 only show higher protein expression in tumour and not normal tissue. ${ }^{29}$ The role of molecular chaperones, such as HSP70 and HSP90, has more recently come to light as an important mechanism of regulation of mutant p53. When p53 binds to HSP90, this inhibits its ability to interact with MDM2, thereby inhibiting its ubiquitination and subsequent degradation. ${ }^{30-32}$ The importance of the interplay between the molecular chaperones and additional ubiquitin ligases such as p300 and CHIP is also becoming more apparent. ${ }^{33,34}$ Alterations in the balance of chaperone-dependent folding of the mutant protein and proteasome-dependent degradation may contribute to the tumour-specific stabilisation observed. ${ }^{22}$ Muller et al. ${ }^{22}$ showed that HSP90 is required to fold mutants into the native conformation or, alternatively, forms a stable complex with mutants that adopt an unfolded conformation. This protects them from binding to HSP70 and subsequent CHIP-dependent degradation. HSP90 has been shown to be highly active in tumours cells ${ }^{35}$ and the relative levels of HSP90 and HSP70 may help to explain some of the heterogeneous expression patterns of mutant p53.

A key step in the HSP90-dependent stabilisation of mutant p53 is the transfer of the native or denatured p53 protein from HSP70 to HSP90. This is facilitated by cochaperones including HOP. ${ }^{36}$ HOP contains multiple tetratricopeptide repeat (TPR) domains that allow it, and other proteins with this motif, to interact with the C terminus of HSP90 and HSP70 to facilitate client protein transfer. S100A2 has been shown to bind to the TPR domain of HOP, resulting in the inhibition of HOP-HSP70 and HOP-HSP90 interactions. ${ }^{37}$ This means that $\mathrm{S} 100 \mathrm{~A} 2$ regulates the key step in stabilisation of mutant p53 by modulating the transfer of the protein from HSP70 to HSP90 and thus protecting it from HSP70/CHIP-dependent degradation. Indeed, our results show S100A2 modulating the levels of mutant protein bound to HSP90. More recently, S100A2 has also been shown to regulate CHIP itself. ${ }^{38}$ This study, however, implies that loss of S100A2 would lead to increased degradation of mutant p53, which contradicts both our findings and the role for S100A2 as a tumour suppressor.

We have shown that S100A2 has an important role in regulating the proliferation of a range of breast cancer cell lines through regulation of the mutant p53/p63 signalling axis, which will further impact on the aggressiveness and metastatic potential of tumours. ${ }^{6,39}$ However, the ability of S100A2 to regulate mutant p53 levels may also impact on the pathology of tumours. When the phenotypes of mice expressing varying amounts of $\mathrm{p} 53 \mathrm{R} 172 \mathrm{H}$ in the same genetic background were compared, it was evident that mutant p53 expression levels influenced the type of tumour. ${ }^{40}$ Low and intermediate levels were associated with predominately sarcomas and some lymphomas and carcinomas. The highest expression was associated with mostly lymphoma, half the frequency of sarcoma and no carcinoma. ${ }^{40}$

We have shown that loss of S100A2 leads to an increase in sensitivity to the HSP90 inhibitor 17-AAG. This may therefore represent a novel therapeutic strategy for targeting S100A2deficient tumours, which according to our studies would include BRCA1-deficient breast cancers. A recent study has shown that BRCA1-deficient cells are acutely sensitive to 17-AAG owing to impaired G2/M checkpoint activation and thus mitotic catastrophe. ${ }^{41}$ They also show that BRCA1 is a client protein of HSP90 and treatment with 17-AAG results in ubiquitination-dependent degradation of BRCA1, resulting in decreased DNA damage repair. ${ }^{41}$ 17-AAG may therefore allow induction of a 'BRCAness' state, which increases sensitivity to common DNA-damaging therapeutics such as 
ionising radiation as well as PARP inhibitors. Our work further highlights the interplay between BRCA1 and HSP90 inhibition. We propose that BRCA1-deficient breast tumours, where abrogation of p53 function through mutation, appear to be a prerequisite for BRCA1-defective cancers to progress, would be extremely sensitive to 17-AAG treatment owing to the loss of S100A2 expression and thus increased HSP90-dependent stabilisation of mutant p53. In addition, 17-AAG may further downregulate BRCA1 levels, and thus S100A2, resulting in greater drug sensitivity.

HSP90 is an attractive drug target owing to the fact that most inhibitors display selectivity for tumour over normal cells. Currently, there are 17 different HSP90 inhibitors in clinical trials in a variety of different cancer types. ${ }^{42}$ However, no HSP90 inhibitor has been FDA approved to date. Although phase I clinical trials of geldanamycin derivatives showed promising results, monotherapy showed little activity in phase II. This may be due to the suboptimal doses of the drugs used to avoid toxicity. ${ }^{43}$ However, HSP90 inhibitors have shown promising results when used in combination with other agents such as herceptin for the treatment of Her2positive breast cancers. ${ }^{44}$ As with most modern targeted therapies, personalising treatments to match the patients' genetic profile or particular tumour types may increase the effectiveness of HSP90 inhibition. S100A2 may therefore represent a novel biomarker of response to HSP90 inhibitors and BRCA1-deficient tumours may benefit from the combination of HSP90 inhibition with DNA-damaging agents such as PARP inhibitors. Alternatively, other agents, which have been shown to interfere with HSP90 binding to client proteins, such as histone deacteylase inhibitors, ${ }^{45}$ may also prove useful in treating BRCA1-deficient cancers.

\section{Materials and Methods}

Cell lines. All cell lines were purchased from ATCC (in partnership with LGC Standards, Middlesex, UK). Cell lines were characterised by isoenzyme/ cytochrome $c$ oxidase I assay and short tandem repeat analysis by the cell bank. Full details of the HCC-EV/BR, MDA468-EV/BR and MCF7 cell lines are provided in Mullan et al. ${ }^{46}$ and Tkocz et al. ${ }^{47}$ HCC1937 and MDA468-EV and $-\mathrm{S} 100 \mathrm{~A} 2$ cells were generated by stable retrovirus transfection of the cells with (i) pBabe EV or pBabe S100A2 constructs, respectively. Infected cells were selected in the presence of $1 \mu \mathrm{g} / \mu \mathrm{l}$ puromycin. All other cell lines were maintained as described previously. ${ }^{48}$

Cloning of S100A2 and its promoter. Flag-tagged S100A2 was PCR amplified and cloned into pBabe-puro EV. Primers are listed in Supplementary Materials and Methods. S100A2 regulatory regions were cloned into pGL3 basic using primers described elsewhere. ${ }^{14}$

Short interfering RNA. Transfections were carried out using Oligofectamine reagent (Invitrogen, Paisley, UK), as outlined in the manufacturer's instructions. SiRNA oligonucleotide were obtained from Dharmacon (Leicestershire, UK) and used at a final concentration of $100 \mathrm{nM}$. siRNA sequences are S100A2 no. 2 , 5'-ACAAGTTCAAGCTGAGTAA-3'; S100A2 no. 3, 5'-GCCAAGAGGGCGACAA GTT-3'. All other siRNA has been described previously. ${ }^{5}$

Western blot analysis. Protein lysates were extracted in EDTA lysis buffer $(0.25 \mathrm{M} \mathrm{NaCl}, 0.1 \%$ IEPGAL, $0.25 \mathrm{M}$ HEPES, $5 \mathrm{mM}$ EDTA, $0.5 \mathrm{mM}$ DTT), separated on a SDS-PAGE gel and then transferred to a PVDF membrane followed by immunoblotting.

Immunoprecipitation. Total, native and denatured p53 was immunoprecipitated from cells using DO-1 (Santa Cruz Biotechnology, Dallas, TX, USA), PAb1620 and PAb240, respectively. Briefly $750 \mu \mathrm{g}$ of proteins were incubated with $2 \mu \mathrm{g}$ antibody coupled to Protein G Dynabeads (Invitrogen) overnight. Samples were washed five times in IP buffer (0.5\% NP-40, 50 mM HEPES (pH 7.5), $100 \mathrm{mM} \mathrm{NaCl}, 1 \mathrm{mM}$ DTT) and resolved by western blot analysis. Densitometry was carried out using ImageJ. ${ }^{49}$

RNA extraction, reverse transcription and real-time quantitative PCR. RNA was extracted using RNA STAT-60 Total RNA extraction Reagent (Tel-Test Inc., Friendswood, TX, USA), reverse transcribed using the Transcriptor First Strand cDNA Synthesis kit (Roche, Burgess Hill, UK) and real-time quantitative PCR analysis performed on the Light Cycler 480 system (Roche) using SYBR Green (Roche) according to the manufacturer's instructions. Primers used are: S100A2 - F, 5'-GCTCTCCTTCCTGGGTCTGT-3' and R, 5'-TGCTCCA GAGAACTGCACAT-3'; p53 - F, 5'-TCCGAGTGGAAGGAAATTTGC-3' and R, 5'-GATGGTGGTACAGTCAGAGCC-3'; cloning full-length Flag-tagged S100A2 - F, 5'-GCCATGGACTACAAGGACGACGATGACAAGATGTGCAGTTCTCTGGAG-3' and $\mathrm{R}, 5^{\prime}$-GCTTCAGGGTCGGTCTG-3'. All other primers have been described previously. ${ }^{5,6,39,50}$

Luciferase assays. Cells were co-transfected with the relevant luciferase constructs and Renilla using GeneJuice (Novagen, Middlesex, UK) according to the manufacturer's instructions. After $24 \mathrm{~h}$, cells were lysed with Passive Lysis Buffer (Promega, Madison, WI, USA) and luciferase and Renilla activities were assessed by luminescence using D-luciferin and coelenterazine as substrates, respectively. Site-directed mutagenesis was carried out using KOD polymerase (Novagen) according to the manufacturer's instructions.

ChIP assays. ChIP assays were performed as described previously. ${ }^{51}$ PCR was performed on extracted DNA using Taq DNA polymerase (Roche) according to the manufacturer's instructions. Primers used are as in Kirschner et al. ${ }^{15}$

HSP90 inhibition. For dose-response curves, cells treated with a range of concentrations of $17-\mathrm{AAG}\left(\log 10^{-12}-\log 10^{-6} \mathrm{M}\right)$ for $72 \mathrm{~h}$ before cell viability was assessed by 3-(4,5-dimethylthiazol-2-yl)-2,5-diphenyltetrazolium bromide (MTT) (Sigma, Dorset, UK). For other studies, cells were incubated with $4 \mu \mathrm{M}$ (MDA-MB-468) or $2 \mu \mathrm{M}$ (HCC1937) 17-AAG for $16 \mathrm{~h}$.

S100A2 expression in breast tumours. Publically available data sets ${ }^{52-54}$ with BRCA1 mutation/methylation status were analysed using Oncomine (www.oncomine.org) for S100A2 expression.

Correlation of S100A2 expression and 17-DMAG IC $_{50}$ values. 17-DMAG IC 50 values for a range of cell lines were extrapolated from Lehmann et al. ${ }^{24}$ Using the publically available data sets used within this paper (GSE10890 and E-TABM-157), microarray data from cell lines was analysed for S100A2 expression using R (www.r-project.org). Samples were background-corrected, normalised and transformed using the Affy package, justRMA (www.bioconductor.org). The probe set for S100A2 was identified and the relevant expression levels determined. Correlation between $\mathrm{S} 100 \mathrm{~A} 2$ and $17-\mathrm{DMAG} \mathrm{IC}_{50}$ values was assessed by Spearman's correlation in Prism.

BrdU incorporation. Cells were treated with $10 \mu \mathrm{M}$ BrdU for 20 min minutes before fixing with $4 \%$ paraformaldehyde. Cells were then stained with the FITC mouse anti-BrdU set (BD Pharmingen, Oxford, UK) according to the manufacturer's instructions. BrdU-positive cells were then counted and expressed as a percentage of total cells.

\section{Conflict of Interest}

The authors declare no conflict on interest.

Acknowledgements. We thank Jean-Christophe Bourdon (University of Dundee) for his kind gift of the PAb240 and PAb1620 antibodies.

1. Miki Y, Swensen J, Shattuck-Eidens D, Futreal PA, Harshman K, Tavtigian S et al. A strong candidate for the breast and ovarian cancer susceptibility gene BRCA1. Science 1994; 266: $66-71$.

2. King MC, Marks JH, Mandell JB. Breast and ovarian cancer risks due to inherited mutations in BRCA1 and BRCA2. Science 2003; 302: 643-646. 
3. Yang Q, Sakurai T, Mori lea. Prognostic significance of BRCA1 expression in Japanese sporadic breast carcinomas. Cancer 2001; 92: 54-60.

4. Mullan PB, Quinn JE, Harkin DP. The role of BRCA1 in transcriptional regulation and cell cycle control. Oncogene 2006; 25: 5854-5863.

5. Buckley NE, Conlon SJ, Jirstrom K, Kay EW, Crawford NT, O'Grady A et al. The \{Delta\}np63 proteins are key allies of BRCA1 in the prevention of basal-like breast cancer. Cancer Res 2011; 71: 1933-1944.

6. Adorno M, Cordenonsi M, Montagner M, Dupont S, Wong C, Hann B et al. A mutant-p53/ Smad complex opposes p63 to empower TGFbeta-induced metastasis. Cell 2009; 137: 87-98.

7. Donato R. Intracellular and extracellular roles of S100 proteins. Microsc Res Tech 2003; 60: $540-551$.

8. Cross SS, Hamdy FC, Deloulme JC, Rehman I. Expression of S100 proteins in normal human tissues and common cancers using tissue microarrays: S100A6, S100A8, S100A9 and S100A11 are all overexpressed in common cancers. Histopathology 2005; 46: 256-269.

9. Wicki R, Franz C, Scholl FA, Heizmann CW, Schafer BW. Repression of the candidate tumor suppressor gene S100A2 in breast cancer is mediated by site-specific hypermethylation. Cell Calcium 1997; 22: 243-254.

10. Liu D, Rudland PS, Sibson DR, Platt-Higgins A, Barraclough R. Expression of calciumbinding protein S100A2 in breast lesions. Br J Cancer 2000; 83: 1473-1479.

11. Lee SW, Tomasetto C, Swisshelm K, Keyomarsi K, Sager R. Down-regulation of a member of the $S 100$ gene family in mammary carcinoma cells and reexpression by azadeoxycytidine treatment. Proc Natl Acad Sci USA 1992; 89: 2504-2508.

12. van Dieck J, Teufel DP, Jaulent AM, Fernandez-Fernandez MR, Rutherford TJ, WyslouchCieszynska A et al. Posttranslational modifications affect the interaction of $\mathrm{S} 100$ proteins with tumor suppressor p53. J Mol Biol 2009; 394: 922-930.

13. Mueller A, Schafer BW, Ferrari S, Weibel M, Makek M, Hochli M et al. The calcium-binding protein S100A2 interacts with p53 and modulates its transcriptional activity. J Biol Chem 2005; 280: 29186-29193.

14. Lapi E, lovino A, Fontemaggi G, Soliera AR, lacovelli S, Sacchi A et al. S100A2 gene is a direct transcriptional target of p53 homologues during keratinocyte differentiation. Oncogene 2006; 25: 3628-3637.

15. Kirschner RD, Sanger K, Muller GA, Engeland K. Transcriptional activation of the tumor suppressor and differentiation gene S100A2 by a novel p63-binding site. Nucleic Acids Res 2008; 36: 2969-2980

16. Boni R, Burg G, Doguoglu A, Ilg EC, Schafer BW, Muller B et al. Immunohistochemical localization of the $\mathrm{Ca}^{2+}$ binding $\mathrm{S} 100$ proteins in normal human skin and melanocytic lesions. Br J Dermatol 1997; 137: 39-43.

17. Shrestha P, Muramatsu Y, Kudeken W, Mori M, Takai Y, Ilg EC et al. Localization of $\mathrm{Ca}(2+)$-binding $\mathrm{S} 100$ proteins in epithelial tumours of the skin. Virch Arch 1998; 432: 53-59.

18. Romano RA, Birkaya B, Sinha S. A functional enhancer of keratin14 is a direct transcriptional target of deltaNp63. J Invest Dermatol 2007; 127: 1175-1186.

19. Tan M, Heizmann CW, Guan K, Schafer BW, Sun Y. Transcriptional activation of the human S100A2 promoter by wild-type p53. FEBS Lett 1999; 445: 265-268.

20. Tsai WC, Tsai ST, Jin YT, Wu LW. Cyclooxygenase-2 is involved in S100A2-mediated tumor suppression in squamous cell carcinoma. Mol Cancer Res 2006; 4: 539-547.

21. Antonini D, Rossi B, Han R, Minichiello A, Di Palma T, Corrado M et al. An autoregulatory loop directs the tissue-specific expression of p63 through a long-range evolutionarily conserved enhancer. Mol Cell Biol 2006; 26: 3308-3318.

22. Muller P, Hrstka R, Coomber D, Lane DP, Vojtesek B. Chaperone-dependent stabilization and degradation of p53 mutants. Oncogene 2008; 27: 3371-3383.

23. Shimamoto S, Kubota Y, Tokumitsu H, Kobayashi R. S100 proteins regulate the interaction of $\mathrm{Hsp} 90$ with cyclophilin 40 and FKBP52 through their tetratricopeptide repeats. FEBS Lett 2010; 584: 1119-1125

24. Lehmann BD, Bauer JA, Chen X, Sanders ME, Chakravarthy AB, Shyr $Y$ et al. Identification of human triple-negative breast cancer subtypes and preclinical models for selection of targeted therapies. J Clin Invest 2011; 121: 2750-2767.

25. Kennedy RD, Gorski JJ, Quinn JE, Stewart GE, James CR, Moore S et al. BRCA1 and c-Myc associate to transcriptionally repress psoriasin, a DNA damage-inducible gene. Cancer Res 2005; 65: 10265-10272.

26. Petitjean A, Mathe E, Kato S, Ishioka C, Tavtigian SV, Hainaut $P$ et al. Impact of mutant p53 functional properties on TP53 mutation patterns and tumor phenotype: lessons from recent developments in the IARC TP53 database. Hum Mutat 2007; 28: 622-629.

27. Terzian T, Suh YA, Iwakuma T, Post SM, Neumann M, Lang GA et al. The inherent instability of mutant p53 is alleviated by Mdm2 or p16INK4a loss. Genes Dev 2008; 22: 1337-1344.

28. Lee KC, Goh WL, Xu M, Kua N, Lunny D, Wong JS et al. Detection of the p53 response in zebrafish embryos using new monoclonal antibodies. Oncogene 2008; 27: 629-640.

29. Lang GA, Iwakuma T, Suh YA, Liu G, Rao VA, Parant JM et al. Gain of function of a p53 hot spot mutation in a mouse model of Li-Fraumeni syndrome. Cell 2004; 119: 861-872.

30. Nagata Y, Anan T, Yoshida T, Mizukami T, Taya Y, Fujiwara T et al. The stabilization mechanism of mutant-type p53 by impaired ubiquitination: the loss of wild-type p53 function and the hsp90 association. Oncogene 1999; 18: 6037-6049.

31. Peng Y, Chen L, Li C, Lu W, Agrawal S, Chen J. Stabilization of the MDM2 oncoprotein by mutant p53. J Biol Chem 2001; 276: 6874-6878.
32. Peng Y, Chen L, Li C, Lu W, Chen J. Inhibition of MDM2 by hsp90 contributes to mutant p53 stabilization. J Biol Chem 2001; 276: 40583-40590.

33. Grossman SR, Deato ME, Brignone C, Chan HM, Kung AL, Tagami H et al. Polyubiquitination of p53 by a ubiquitin ligase activity of p300. Science 2003; 300 : 342-344.

34. Esser C, Scheffner M, Hohfeld J. The chaperone-associated ubiquitin ligase CHIP is able to target p53 for proteasomal degradation. J Biol Chem 2005; 280: 27443-27448.

35. Kamal A, Thao L, Sensintaffar J, Zhang L, Boehm MF, Fritz LC et al. A high-affinity conformation of Hsp90 confers tumour selectivity on Hsp90 inhibitors. Nature 2003; 425: 407-410.

36. King FW, Wawrzynow A, Hohfeld J, Zylicz M. Co-chaperones Bag-1, Hop and Hsp40 regulate $\mathrm{Hsc70}$ and $\mathrm{Hsp90}$ interactions with wild-type or mutant p53. EMBO J 2001; 20: 6297-6305.

37. Shimamoto S, Takata M, Tokuda M, Oohira F, Tokumitsu H, Kobayashi R. Interactions of S100A2 and S100A6 with the tetratricopeptide repeat proteins, Hsp90/Hsp70-organizing protein and kinesin light chain. J Biol Chem 2008; 283: 28246-28258.

38. Shimamoto S, Kubota Y, Yamaguchi F, Tokumitsu H, Kobayashi R. $\mathrm{Ca}^{2+} / \mathrm{S} 100$ proteins act as upstream regulators of the chaperone-associated ubiquitin ligase $\mathrm{CHIP}(\mathrm{C}$ terminus of Hsc70-interacting protein). J Biol Chem 2013; 288: 7158-7168.

39. Girardini JE, Napoli M, Piazza S, Rustighi A, Marotta C, Radaelli E et al. A Pin1/mutant p53 axis promotes aggressiveness in breast cancer. Cancer Cell 2011; 20: 79-91.

40. Goh AM, Coffill CR, Lane DP. The role of mutant p53 in human cancer. J Pathol 2011; 223 $116-126$.

41. Stecklein SR, Kumaraswamy E, Behbod F, Wang W, Chaguturu V, Harlan-Williams LM et al. BRCA1 and HSP90 cooperate in homologous and non-homologous DNA doublestrand-break repair and G2/M checkpoint activation. Proc Natl Acad Sci USA 2012; 109: 13650-13655.

42. Barrott JJ, Haystead TA. Hsp90, an unlikely ally in the war on cancer. FEBS J 2013; 280: 1381-1396.

43. Usmani SZ, Bona R, Li Z. 17 AAG for HSP90 inhibition in cancer - from bench to bedside. Curr Mol Med 2009; 9: 654-664.

44. Modi S, Stopeck A, Linden H, Solit D, Chandarlapaty S, Rosen N et al. HSP90 inhibition is effective in breast cancer: a phase II trial of tanespimycin (17-AAG) plus trastuzumab in patients with HER2-positive metastatic breast cancer progressing on trastuzumab. Clin Cancer Res 2011; 17: 5132-5139.

45. Bali P, Pranpat M, Bradner J, Balasis M, Fiskus W, Guo F et al. Inhibition of histone deacetylase 6 acetylates and disrupts the chaperone function of heat shock protein 90: a novel basis for antileukemia activity of histone deacetylase inhibitors. J Biol Chem 2005: 280: 26729-26734.

46. Mullan PB, Hosey AM, Buckley NE, Quinn JE, Kennedy RD, Johnston PG et al. The 2,5 oligoadenylate synthetase/RNaseL pathway is a novel effector of BRCA1- and interferon-gamma-mediated apoptosis. Oncogene 2005; 24: 5492-5501.

47. Tkocz D, Crawford NT, Buckley NE, Berry FB, Kennedy RD, Gorski JJ et al. BRCA1 and GATA3 corepress FOXC1 to inhibit the pathogenesis of basal-like breast cancers. Oncogene 2011; 31: 3667-3678.

48. Neve RM, Chin K, Fridlyand J, Yeh J, Baehner FL, Fevr T et al. A collection of breast cancer cell lines for the study of functionally distinct cancer subtypes. Cancer Cell 2006; 10 : 515-527.

49. Schneider CA, Rasband WS, Eliceiri KW. NIH Image to ImageJ: 25 years of image analysis. Nat Methods 2012; 9: 671-675.

50. Buckley NE, Nic An Tsaoir CB, Blayney JK, Oram LC, Crawford NT, D'Costa ZC et al. $B R C A 1$ is a key regulator of breast differentiation through activation of Notch signalling with implications for anti-endocrine treatment of breast cancers. Nucleic Acids Res 2013; 41: 8601-8614.

51. Hosey AM, Gorski JJ, Murray MM, Quinn JE, Chung WY, Stewart GE et al. Molecular basis for estrogen receptor alpha deficiency in BRCA1-linked breast cancer. J Natl Cancer Inst 2007; 99: 1683-1694.

52. Waddell N, Cocciardi S, Johnson J, Healey S, Marsh A, Riley J et al. Gene expression profiling of formalin-fixed, paraffin-embedded familial breast tumours using the whole genome-DASL assay. J Pathol 2010; 221: 452-461.

53. Richardson AL, Wang ZC, De Nicolo A, Lu X, Brown M, Miron A et al. X chromosomal abnormalities in basal-like human breast cancer. Cancer Cell 2006; 9: 121-132.

54. Hedenfalk I, Duggan D, Chen Y, Radmacher M, Bittner M, Simon R et al. Gene-expression profiles in hereditary breast cancer. N Engl J Med 2001; 344: 539-548.

(c) (i) () $\Theta$ Cell Death and Disease is an open-access journal (c) licensed under a Creative Commons Attribution-NonCommercialNoDerivs 3.0 Unported License. To view a copy of this license, visit http://creativecommons.org/licenses/by-nc-nd/3.0/ 